\title{
miR-1207-5p and miR-1266 suppress gastric cancer growth and invasion by targeting telomerase reverse transcriptase
}

\author{
L Chen ${ }^{1,4}$, M-H Lü ${ }^{1,4}$, D Zhang ${ }^{1}$, N-B Hao ${ }^{1}$, Y-H Fan ${ }^{1}$, Y-Y Wu' ${ }^{1}$, S-M Wang ${ }^{1}$, R Xie ${ }^{1}$, D-C Fang ${ }^{2}$, H Zhang ${ }^{3}$, C-J Hu ${ }^{*, 1}$ and S-M Yang ${ }^{\star, 1}$
}

hTERT is the catalytic subunit of the telomerase complex. Elevated expression of hTERT is associated with the expansion and metastasis of gastric tumor. In this study, we aimed to identify novel tumor suppressor miRNAs that restrain hTERT expression. We began our screen for hTERT-targeting miRNAs with a miRNA microarray. miRNA candidates were further filtered by bioinformatic analysis, general expression pattern in different cell lines, gain-of-function effects on hTERT protein and the potential of these effects to suppress hTERT $3^{\prime}$ untranslated region ( $3^{\prime}$ UTR) luciferase activity. The clinical relevance of two miRNAs (miR-1207-5p and miR-1266) was evaluated by real-time RT-PCR. The effects of these miRNAs on cell growth, cell cycle and invasion of gastric cancer cells were measured with CCK-8, flow cytometry and transwell assays. Finally, the ability of these miRNAs to suppress the transplanted tumors was also investigated. Fourteen miRNAs were identified using a combination of bioinformatics and miRNA microarray analysis. Of these fourteen miRNAs, nine were expressed at significantly lower levels in hTERT-positive cell lines compared with hTERT-negative cell lines and five could downregulate hTERT protein expression. Only miR-1207-5p and miR-1266 interacted with the $3^{\prime}$ UTR of hTERT and the expression levels of these two miRNAs were significantly decreased in gastric cancer tissues. These two miRNAs also inhibited gastric tumor growth in vitro and in vivo. Altogether, miR1207-5p and miR-1266 were determined to be hTERT suppressors in gastric cancer, and the delivery of these two miRNAs represents a novel therapeutic strategy for gastric cancer treatment.

Cell Death and Disease (2014) 5, e1034; doi:10.1038/cddis.2013.553; published online 30 January 2014

Subject Category: Cancer

Telomeres lie at the ends of linear eukaryotic chromosomes and are composed of non-coding tandem repeated sequences. ${ }^{1}$ As DNA polymerase can only synthesize DNA in the $5^{\prime}$ to $3^{\prime}$ direction and the short RNA primers used during conventional DNA replication are lost, the lagging DNA strand is incompletely replicated and the telomere length decreases with each cell division. Human telomerase reverse transcriptase (hTERT), the catalytic subunit of telomerase, can reverse telomere shortening. As normal proliferating cells enter into the process of apoptosis or necrosis, the expression of hTERT expression gradually decreases and cells cease proliferation. ${ }^{2}$ However, elevated hTERT expression and the activation of telomerase can result in unlimited proliferative ability in tumor cells. ${ }^{3}$ Studies have shown that hTERT has an important role in cancer tumorigenesis, growth, migration and invasion. ${ }^{4}$ In our previous studies, we found that hTERT expression is positively correlated with many clinicopathological variables, including tumor location, size, depth of invasion, histological grade, lymph node metastasis, TNM stage and distance of metastasis. ${ }^{5}$ Therefore, hTERT is considered as an attractive therapeutic target for cancer treatment, and understanding the regulatory mechanisms controlling hTERT expression is of great interest.

To date, most research probing the regulation of hTERT has been focused on gene transcription. Sequence analysis has revealed that the hTERT promoter is GC-rich, lacks TATA and CAAT boxes, and contains binding sites for various transcription factors, including c-Myc, AP-1 and E2F and the tumor suppressors WT1 and p53. ${ }^{6}$ However, accumulating evidence has indicated that post-transcriptional regulation, specifically microRNA (miRNA)-mediated regulation, represents an additional level of control that fine-tune protein expression levels across multiple oncogenic signaling pathways. Consequently, the dysregulation of miRNAs is often detrimental to cells and can contribute to the development of human diseases, including cancer. Recently, the delivery of miRNA mimics or inhibitors has emerged as a promising strategy for cancer treatment. ${ }^{7}$ Therefore, the identification of the miRNA suppressors regulating hTERT expression and examination of their potential use in anti-tumor therapies is crucial.

Through miRNA expression profiling with hTERT-positive and hTERT-negative cancer cell lines, as well as bioinformatics

\footnotetext{
${ }^{1}$ Department of Gastroenterology, Xinqiao Hospital, Third Military Medical University, Chongqing 400037, China; ${ }^{2}$ Institute of Gastroenterology, Southwest Hospital, Third Military Medical University, Chongqing 400037, China and ${ }^{3}$ Department of Gastroenterology, The Second Affiliated Hospital, Chongqing Medical University, Chongqing 400037, China

${ }^{*}$ Corresponding author: C-J Hu or S-M Yang, Department of Gastroenterology, Xingiao Hospital, Third Military Medical University, Chongqing 400037, China. Tel: +86 23 68752963; Fax: +86 2368755604 (C-JH) or Tel/Fax: +86 2368755604 (S-MY); E-mail: hcj6699@yahoo.com or shimingyang @yahoo.com

${ }^{4}$ These authors contributed equally to this work.

Keywords: tumor-suppressor miRNA; hTERT; gastric cancer

Abbreviations: hTERT, human telomerase reverse transcriptase; miRNA, microRNA; UTR, untranslated region

Received 17.7.13; revised 25.11.13; accepted 12.12.13; Edited by M Agostini
} 
analyses, we identified miRNAs with the potential to directly regulate $\mathrm{hTERT}$. The clinical association between miRNAs and hTERT was verified within 58 pairs of gastric cancer tissues and corresponding adjacent tissues. Finally, we determined the function of hTERT-targeting miRNAs in vitro and in vivo.

\section{Results}

Identification of fourteen candidate hTERT-targeting miRNAs through expression profiling and bioinformatic analysis. To identify the tumor suppressor miRNAs controlling hTERT expression, we hypothesized that the expression levels of such miRNAs should be restricted in hTERTpositive tissues. hTERT-positive gastric cancer tissues and hTERT-negative corresponding adjacent tissues (Figure 1a) were collected from the same patient undergoing surgical operations. Total RNA was isolated and subjected to miRCURY LNA Array analysis. The results are on the website: http://www.ncbi.nlm.nih.gov/geo/query/acc.cgi?acc= GSE47107; the GEO accession number is GSE47107. With a cutoff set at a twofold change, 210 miRNAs were determined to be significantly downregulated $(P<0.05$, analyzed in triplicates) in gastric cancer tissues compared with corresponding adjacent tissues (Figure 1b, details shown in Supplementary Table 2). Additionally, we scanned the $3^{\prime}$ UTR region of hTERT using the widely used online targetgene prediction software program TargetScan (http:// www.targetscan.org/mamm_31/). As shown in Figure 1c, 57 miRNAs were predicted to have binding potential in the $3^{\prime}$ UTR region of hTERT mRNA (details shown in Supplementary Table 3). Among these 57 miRNAs, 14 expressed at lower level in the hTERT-positive gastric tumor tissue than that of hTERT-negative corresponding adjacent tissue (Figure 1d).

Shortening the candidate list with a general miRNA expression pattern. We reasoned that the restricted expression should be a general pattern for the most critical miRNA regulators of hTERT in various tumor cells. We utilized this rationale to shorten the candidate list generated above by comparing miRNA levels in hTERT-positive (BGC823, MKN28, SGC-7901 and KATO-III) tumor cell lines to hTERT-negative cell lines (U2OS, HF) (Figures $2 a$ and b). The relative quantity (RQ) of the 14 previously identified miRNAs was determined using real-time RT-PCR (Figure 2c). N/P values were determined as the mean miRNA expression level of the two hTERT-negative cell lines compared with the mean expression level of the four hTERTpositive cell lines. Consistent with our earlier findings, in general, the 14 miRNAs that were predicted to target hTERT were downregulated in the hTERT-positive cancer cell lines. We established the selection threshold as N/P ratios $>2$, and as a result, 9 of the 14 miRNAs were determined to be putative hTERT-regulating miRNAs (Figure 2d).

miR-1027-5p and miR-1266 directly regulate hTERT expression. To determine whether these nine miRNAs suppress the expression of endogenous hTERT, western blotting was performed after SGC-7901 cells were transiently transfected (for $48 \mathrm{~h}$ ) with miRNA mimics or together with their corresponding specific inhibitor (antago-miR). As shown in Figure 3a, only five miRNAs (miR-138, miR-491-5p, miR1182, miR-1207-5p and miR-1266) suppressed hTERT expression in SGC-7901 cells compared with the control miRNA (Scramble), while the suppression of hTERT could be partly rescued in the presence of specific antago-miRs. To confirm whether this suppression directly affected hTERT, dual-luciferase reporter assays were conducted to test the potential interaction between hTERT $3^{\prime}$ UTR and these five miRNAs. The reporter assays demonstrated that only miR1207-5p and miR-1266 repressed Luc-hTERT-3'UTR directly and the repression could be partly restored by antago-miR1207-5p or antago-miR-1266, respectively. However, miR-138, miR-491-5p and miR-1182 had no significant effects on luciferase activity in the presence or absence of antago-miR (Figure $3 b$ ). Furthermore, when the predicted targeting site in hTERT 3' UTR was mutated to avoid binding to the 'seed region' (Figure 3c, left panel), miR-1207-5p and miR-1266 were not able to effectively suppress the luciferase expression under the control of the hTERT $3^{\prime}$ UTR. To further confirm that the miRNA inhibited hTERT expression by interacting with the $3^{\prime}$ UTR of hTERT, we performed forcedexpression experiments in SGC-7901 cells with constructs expressing individual miRNAs and hTERT cDNAs lacking the full-length $3^{\prime}$ UTR. Consistent with its suppressive role in controlling the endogenous level of hTERT protein (Figure 3a), miR-1266 and miR-1207-5p were able to significantly inhibit hTERT expression in the presence of its $3^{\prime}$ UTR; in contrast, miR-1266 and miR-1207-5p failed to regulate exogenous hTERT when the hTERT $3^{\prime}$ UTR was missing (Figure 3d). Taken together, these results demonstrate that both miR-1266 and miR-1207-5p are direct miRNA suppressors that restrict hTERT expression.

Expression levels of miR-1207-5p and miR-1266 are clinically relevant. The above experiments clearly demonstrate that the five identified miRNAs repress hTERT expression and, furthermore, that two of these miRNAs can directly bind to the $3^{\prime}$ UTR of hTERT. To explore whether the dysregulation of miR-1207-5p and miR-1266 is involved in tumorigenesis, we compared the expression levels of these miRNAs in gastric cancer tissues and matched corresponding adjacent tissues $(n=58)$. Our data show that miR-1182, $\mathrm{miR}-1207-5 p$ and miR-1266 are significantly repressed in gastric tumor samples $(P<0.05)$ (Figure $4 a)$. We further evaluated the relationship between the expression levels of these miRNAs in the patients' gastric cancer tissues and the survival times of the patients. For each miRNA analysis, the patients were classified as the higher miRNA expression group (relative quantity of miRNA $>$ the average relative quantity of miRNA of all patients) or the lower miRNA expression group (relative quantity of miRNA $\leq$ average relative quantity of miRNA). No significant association was found between miRNA (miR-1207-5p, miR-1266) expression and age or gender (Supplementary Table 4). As shown in Figure 4b, miR-1207-5p expression in gastric cancer tissues does not correlate with the survival time of the patients. Interestingly, higher miR-1266 expression in gastric cancer tissues correlates with a longer patient survival time. 
a

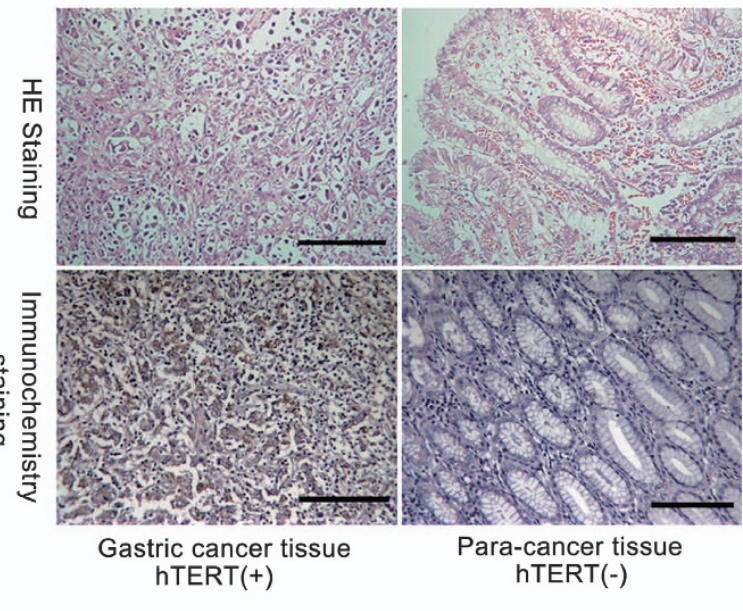

C Prediction of miRNAs targeting hTERT by TargetScan

\begin{tabular}{cccc}
\hline No. & \multicolumn{1}{c}{ miRNA } & Position of hTERT 3'UTR & context score \\
1 & hsa-miR-1266 & $395-401$ & 94 \\
2 & hsa-miR-491-5p & $302-308$ & 93 \\
3 & hsa-miR-491-5p & $235-241$ & 92 \\
4 & hsa-miR-1323 & $547-553$ & 91 \\
5 & hsa-miR-1234 & $18-24$ & 91 \\
6 & hsa-miR-5480 & $547-553$ & 90 \\
7 & hsa-miR-532-3p & $514-520$ & 89 \\
8 & hsa-miR-138 & $33-39$ & 89 \\
9 & hsa-miR-299-3p & $304-310$ & 87 \\
10 & hsa-miR-608 & $258-264$ & 84 \\
$\ldots . .$. & & \\
\hline
\end{tabular}

\section{d}

miR-138
miR-1266
miR-491-5p
miR-1323
miR-299-3p
miR-668
miR-1228
miR-1182
miR-1207-5p
miR-637
miR-654-5p
miR-512-5p
miR-886-3p
miR-1234
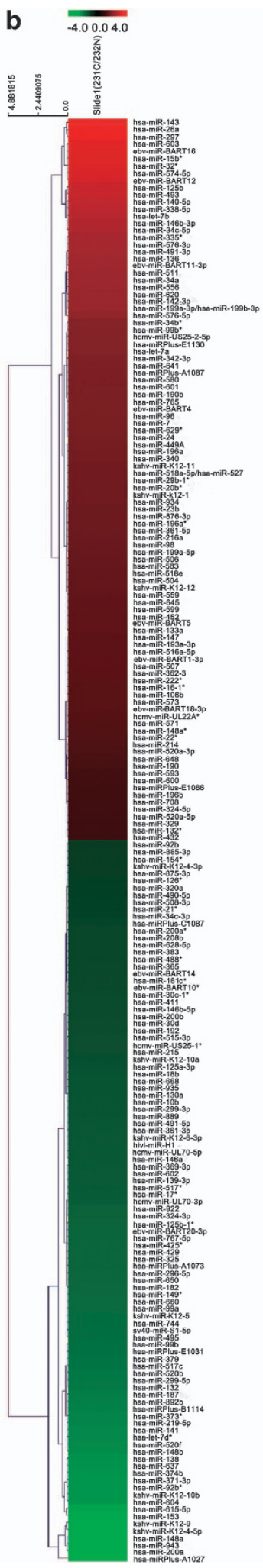

Figure 1 Identification of 14 candidate hTERT-targeting miRNAs through expression profiling and bioinformatic analysis. (a) Immunohistochemistry of hTERT-positive gastric cancer tissues and hTERT-negative corresponding adjacent tissues. (b) Alteration of miRNAs in gastric cancer tissues in comparison to corresponding adjacent tissues as determined by miRNA microarray analysis (the red part represents the up-regulated miRNAs, while the green part represents the downregulated miRNAs). All the miRNAs are listed in Supplementary Table 2. (c) Prediction of miRNAs that have hTERT mRNA 3' UTR region-binding potential by TargetScan (http://www.targetscan.org/mamm_31/). Fifty-seven miRNAs (listed in supplementary table 3) were predicted to have hTERT-binding potential. (d) miRNAs screened from the results of expression profiling (b) and bioinformatic analysis (c) 
a

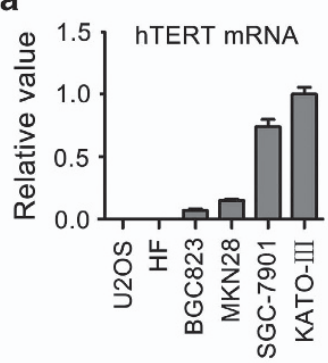

b hTERT negative

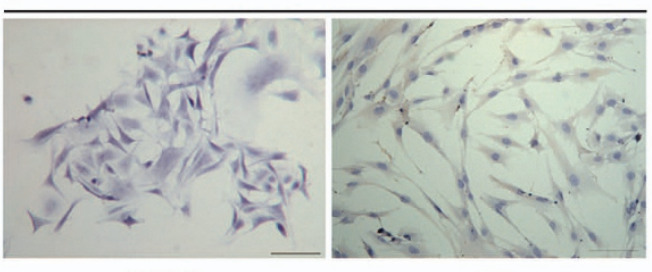

U2OS
$\mathrm{HF}$

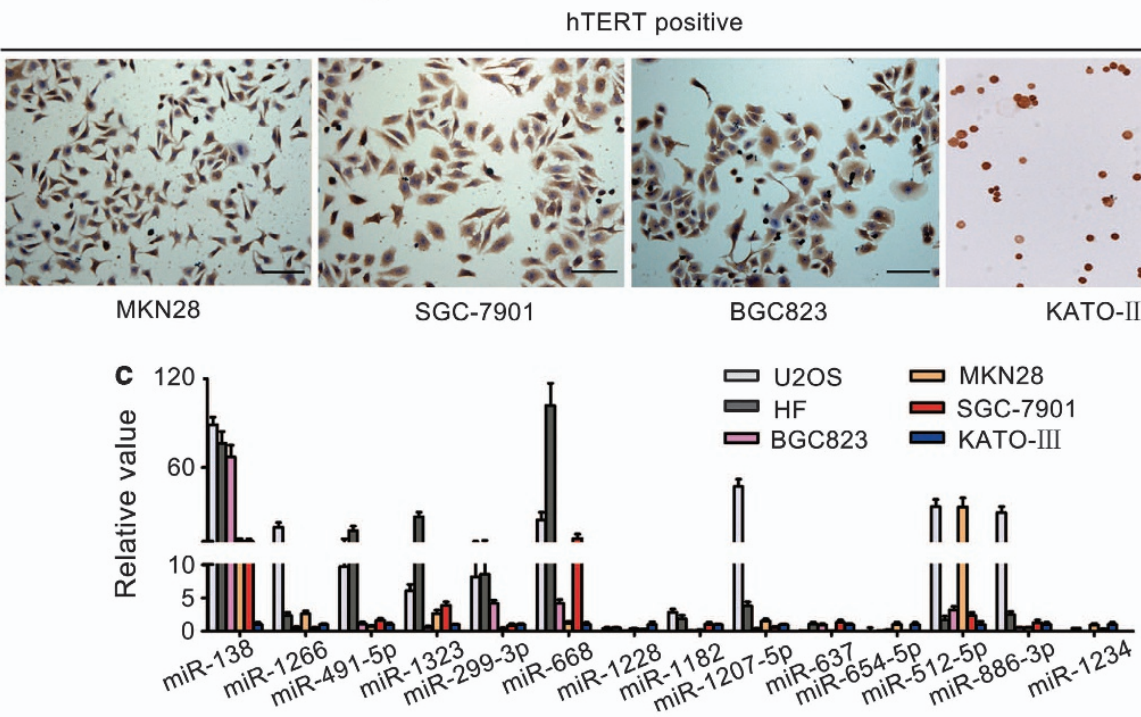

d

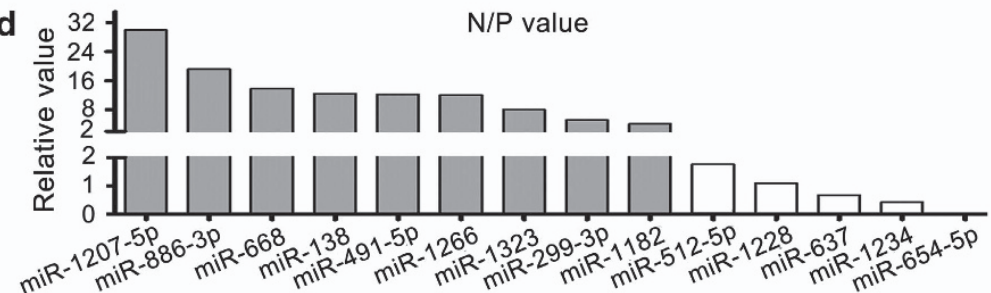

Figure 2 Shortening the candidate list by considering a general miRNA expression pattern. (a) hTERT mRNA expression levels in different cell lines. Real-time RT-PCR was used to detect the expression of hTERT mRNA in U2OS, HF, BGC823, MKN28, SGC-7901 and KATO-III cell lines. (b) Immunohistochemistry of hTERT expression in the above cell lines. Immunohistochemistry was used to further confirm hTERT-negative (U2OS, HF) and hTERT-positive (BGC823, MKN28, SGC-7901 and KATO-III) cell lines. (c) Expression of different miRNAs in the cell lines. Real-time RT-PCR was applied to analyze the expression of the 14 miRNAs in the different cell lines. (d) N/P values of the fourteen miRNAs. N/P values were determined as the mean miRNA expression level in the two hTERT-negative cell lines compared with the mean expression level in the four hTERT-positive cell lines. Nine miRNAs passed the screening process (N/P > 2, shown in black). N, hTERT-negative cell lines; $\mathrm{P}$, hTERT-positive cell lines

miR-1207-5p and miR-1266 function as tumor suppressors in cultured SGC-7901 gastric cancer cells in vitro. The above data suggest that, through the deregulation of hTERT expression, the loss of miR-1207-5p or miR-1266 might be critical for cells to acquire a malignant phenotype; reciprocally, we speculated that reintroduction of miR1207-5p or miR-1266 could interfere with hTERT expression and affect the tumorigenic potential of gastric cancer cells. To test this hypothesis, SGC-7901 cells were infected with lentivirus expressing individual miRNAs. Subsequently, these cells were transfected with pIRES2hTRET or control (pIRES2). As shown in Figure 5a, both miR-1207-5p and miR-1266 inhibited SGC-7901 cell proliferation compared with Scramble.
Flow cytometry revealed that miR-1207-5p and miR-1266 induced cell cycle arrest at the G1-to-S transition (Figure 5b). Furthermore, these miRNAs also significantly suppressed gastric cancer cell invasion (Figure $5 \mathrm{c}$ and d). Interestingly, the above figures also showed that reintroduction of hTERT in SGC-7901 cells partly restored the above phenotype. These results suggest that miR-1207-5p or miR-1266 have anti-tumor roles via hTERT. In addition to miR-1207-5p and miR-1266, three other hTERT-inhibiting miRNAs identified in our screening (miR-138, miR-491-5p and miR-1182) also demonstrated similar suppressive effects on proliferation, the cell cycle and invasion in SGC-7901 cells (Supplementary Figure 3). These results were observed despite the lack of direct interaction between 
a

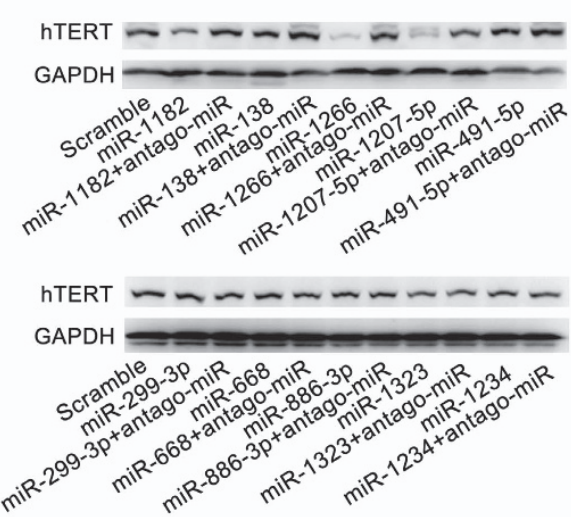

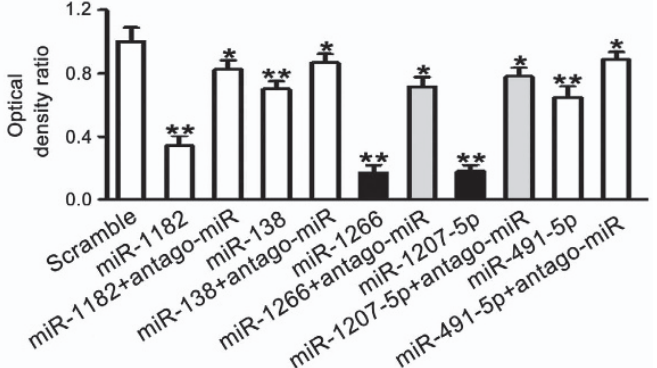

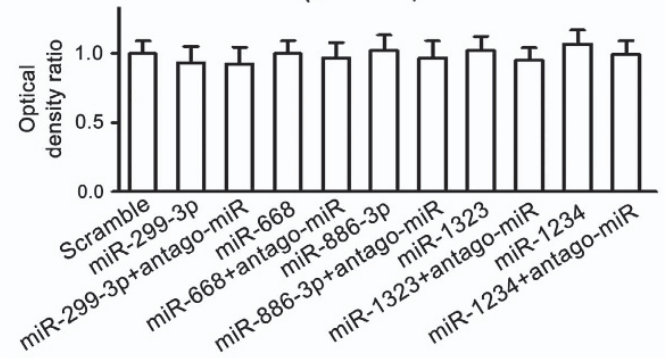

b

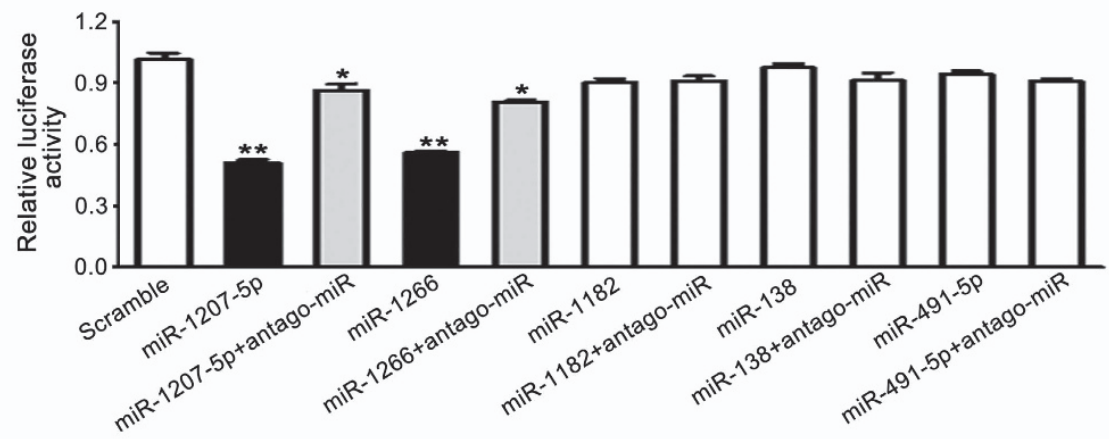

c
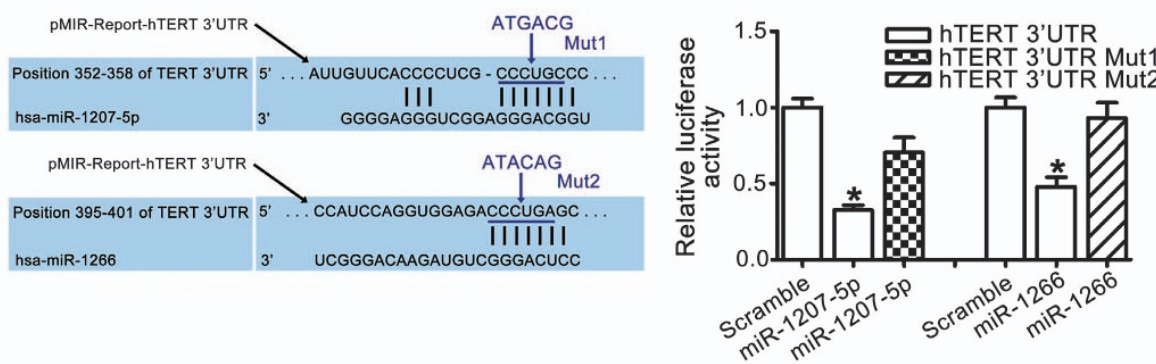

d

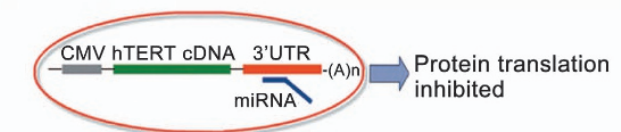

Endogenous hTERT transcriptional region

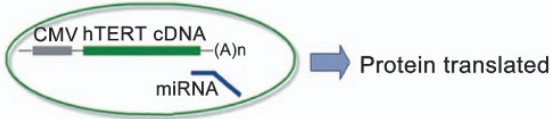

Exogenous pIRES2-hTERT transcriptional region

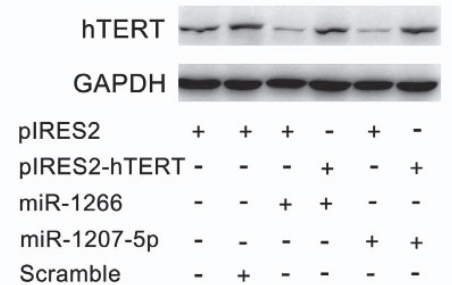

Figure 3 Searching for miRNAs that directly regulate hTERT expression. (a) The effect of the miRNAs on hTERT protein expression. After SGC-7901 cells were transfected with different miRNA mimics or together with their corresponding antago-miRs for $96 \mathrm{~h}$, western blots were performed to test hTERT expression. The results indicated that five miRNAs (miR-138, miR-491-5p, miR-1182, miR-1207-5p and miR-1266) downregulated hTERT expression. (b) Luciferase assays for hTERT 3'UTR. Two miRNAs (miR-1207-5p and miR-1266) were found to reduce luciferase activity, while the reduction could be partly rescued by their specific antago-miRs. (c) Luciferase assays for the wild type and the mutated type of hTERT $3^{\prime}$ UTR. Left panel, the design of mutated sites in hTERT $3^{\prime}$ UTR. Right panel, the effect of the miRNAs on luciferase of hTERT 3' UTR. mut1, hTERT 3' UTR with the mutated miR-1207-5p binding site; mut2, hTERT 3'UTR with the mutated miR-1266 binding site. (d) The effect of the exogenous hTERT to rescue the miRNAs-mediated downregulation of endogenous hTERT. Left panel, the design of the exogenous hTERT expression plasmid. The pIRES2-hTERT plasmid lacks the hTERT $3^{\prime}$ UTR; thus, miRNAs could not bind to the $3^{\prime}$ UTR of the hTERT transcript. Right panel, SGC-7901 cells were transfected with mimics of miRNAs together with the pIRES2 or the pIRES2-hTERT plasmid $\left({ }^{*} P<0.05,{ }^{* *} P<0.01\right.$, one-way ANOVA) 

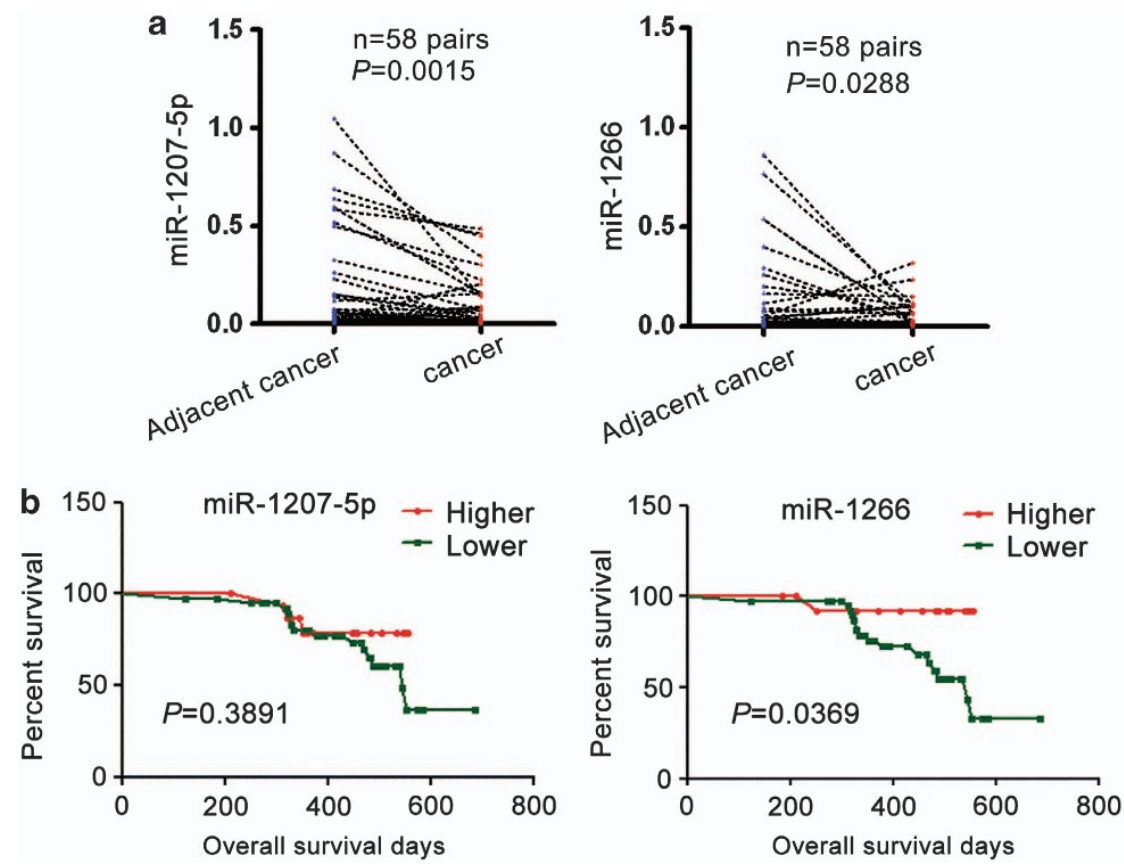

Figure 4 The expression levels of miR-1207-5p and miR-1266 are clinically relevant. Fifty-eight pairs of gastric cancer tissues and matched corresponding adjacent tissues were collected for miRNA expression analysis. Real-time RT-PCR was performed to analyze the expression levels of miR-1207-5p and miR-1266. The housekeeping gene U6 was amplified as an internal reference to adjust for well-to-well variations in RNA input. (a) The miRNA expression levels in cancer tissues and corresponding adjacent tissues of 58 gastric cancer patients. Both miR-1207-5p and miR-1266 were significantly downregulated in gastric cancer tissues. (b) Kaplan-Meier's analysis of overall survival time of gastric cancer patients according to the expression level of miRNAs

these miRNAs and hTERT $3^{\prime}$ UTR. To demonstrate the specific regulation of hTERT by miRNAs, corresponding antago-miRs were also transfected into the stable expression cells. As shown in Figure 6, antago-miRs rescued the phenotype of the corresponding miRNAs on cell proliferation (Figure 6a), cell cycle arrest (Figure 6b) and cell invasion (Figures $6 \mathrm{c}$ and d). Meanwhile, knockdown of hTERT is sufficient to phenotype the effects of the above miRNAs (Supplementary Figure 4A-D)

Both miR-1207-5p and miR-1266 inhibit the growth of transplanted SGC-7901 cells in nude mice. To explore whether miR-1207-5p and miR-1266 could mediate tumor suppression in vivo, suspensions ( $\left.10^{7} \mathrm{pfu} / \mathrm{ml}\right)$ of miR-1207-5p, miR-1266 or the negative control lentivirus vector Scramble were injected into transplanted gastric tumors in nude mice. As shown in Figure $7 a-c, m i R-1207-5 p$ and miR-1266 significantly inhibited the growth of tumors and reduced tumor volume compared with Scramble. This result is quite similar to that of knockdown of hTERT in the xenograft nude mouse (Supplementary Figures 4E-F). The tumors were harvested and subjected to real-time RT-PCR analysis, which showed that the expression levels of both miRNAs were clearly increased in the miR-1207-5p and miR-1266 groups compared with the Scramble group (Figure $7 d$, left panel). Consistently, hTERT expression in the transplanted tumors was clearly downregulated in the miR-1207-5p and miR-1266 groups compared with controls (Figure $7 \mathrm{~d}$, right panel). Moreover, immunochemistry of both PCNA and Ki-67 revealed that the two miRNAs repressed tumor cells proliferation (Figures $7 \mathrm{e}$ and $\mathrm{f}$ ).

\section{Discussion}

In this study, using a combination of miRNA microarrays and bioinformatics, we identified 14 miRNAs that were potentially involved in the regulation of hTERT. Next, we narrowed our focus to nine miRNAs using a general miRNA expression pattern. Of these nine miRNAs, five were able to inhibit hTERT expression, but only miR-1207-5p and miR-1266 could bind to the hTERT $3^{\prime}$ UTR. The expression levels of miR-1207-5p and miR-1266 were significantly decreased in 58 pairs of the gastric cancer tissues compared with their matched corresponding adjacent tissues. We also found that both miR-1207-5p and miR-1266 triggered the downregulation of hTERT expression, suppressed proliferation, decreased invasion and induced cell cycle arrest in gastric cancer cells in vitro. Furthermore, these two miRNAs inhibited the growth of xenograft gastric tumors in nude mice (summarized in Supplementary Figure 1).

Strategies that target hTERT expression in tumors mainly involve the following techniques: antisense genes, ribozymes, inhibitors of reverse transcriptase, nucleoside analogs, chemical drugs and immunotherapy. ${ }^{8}$ Nucleoside analogs, such as base-modified 3'-azido-2', $3^{\prime}$-dideoxynucleoside triphosphate, ${ }^{9}$ Abacavir, ${ }^{10}$ oligonucleotide $\mathrm{n} 3^{\prime} \rightarrow \mathrm{p5}^{\prime}$ phosphoramidates and thio-phoshoramidates have been reported to effectively inhibit TERT and telomerase activity. ${ }^{11}$ Widely used clinical anticancer drugs, such as vindesine, temsirolimus and cyclosporine, were found to be more potent than the standard inhibitor of TERT curcumin. ${ }^{12,13}$ Multiple phase I clinical trials involving hTERT immunotherapy have already been conducted in patients with advanced 
a
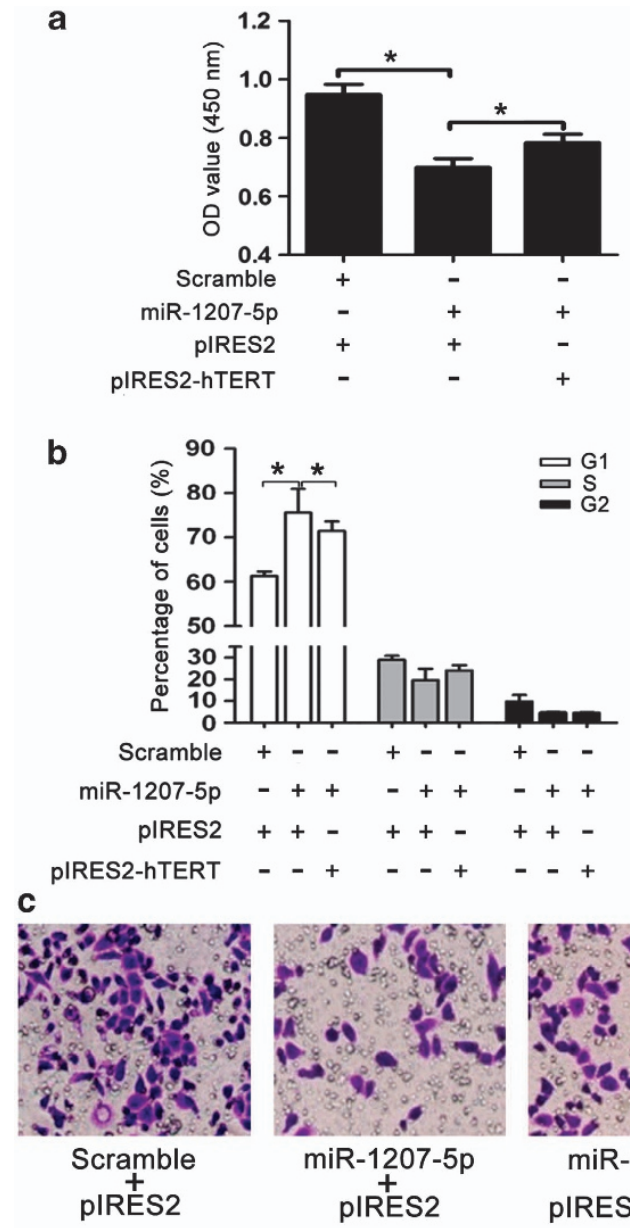

b

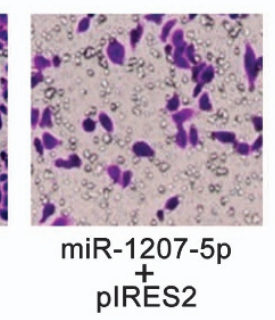

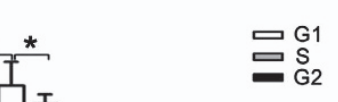
ए $\mathrm{S}_{2}$

\section{d}
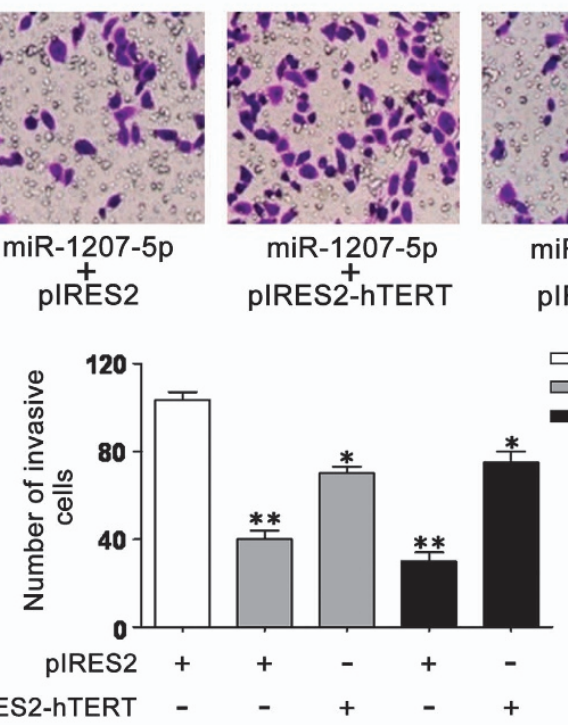

PIRES2-hTERT
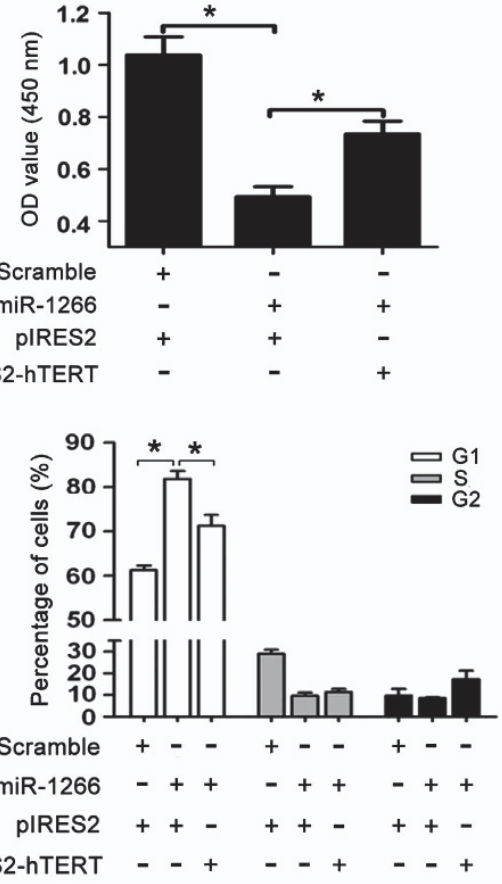
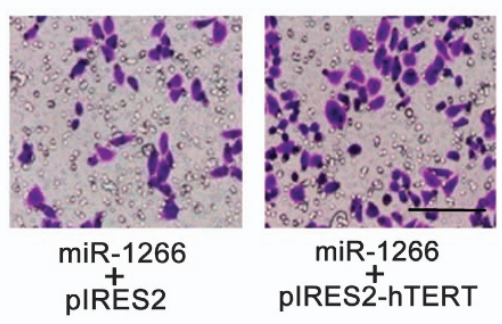

Scramble

$\square \mathrm{miR}-1207-5 \mathrm{p}$

- miR-1266

Figure 5 Biological effects of miR-1207-5p and miR-1266 on gastric cancer cells. SGC-7901 cells were infected with lentivirus packaged with different miRNAs. Each experiment was performed in triplicates. (a) Effects of miRNAs on cell proliferation. After the cells infected with the lentivirus were seeded in 96 wells for $18 \mathrm{~h}$, they are transfected with pIRES2 or pIRES2-hTERT, CCK-8 assays were performed $48 \mathrm{~h}$ later. (b) Effects of miRNAs on cell cycle. Cells were treated the same as (a) and flow cytometry were performed. ( $\mathbf{c}$ and $\mathbf{d}$ ) Effects of miRNAs on the invasion of gastric cancer cells. Cells were treated the same as in (a) and then they were seeded into the Matrigel invasion chamber. Subsequently, the chambers were transferred to wells filled with culture medium containing $10 \%$ FBS. After $48 \mathrm{~h}$ of incubation, the number of invasive cells from five random areas of the membrane was counted using light microscopy. Both miR-1207-5p and miR-1266 significantly suppressed cell invasion. The mean of triplicate experiments \pm S.D. is shown $\left({ }^{\star} P<0.05\right.$, ${ }^{*} P<0.01$, one-way ANOVA)

cancer and involved the injection of 1540 and E611 hTERT peptides. ${ }^{14,15}$ In a previous study, we found that exogenous antisense hTERT can inhibit proliferation and partially reverse malignant phenotypes in SGC-7901 cells by suppressing telomerase activity. ${ }^{16}$ Furthermore, the transduction of dendritic cells with recombinant-adenovirus hTERT induced hTERTspecific cytotoxic T lymphocytes in vitro and in vivo. ${ }^{17,18}$ During a previous study, we showed that hTERT promoted the invasion of telomerase-negative tumor cells in vitro. ${ }^{19}$ Moreover, we also demonstrated that hTERT RNAi overcame the resistance of HCC cells to TRAIL via the mitochondrial type II apoptosis pathway and a telomerase-dependent pathway. ${ }^{20}$ To provide a valuable noninvasive method to evaluate the real-time therapeutic response of tumors in vivo, we constructed a lentivirus system in which an optimized hTERT promoter is used to drive expression of the cytosine deaminase (CD) gene, allowing telomerase-positive tumors to be visualized and their growth to be repressed following intra-tumoral injection of the lentivirus. ${ }^{21}$ 


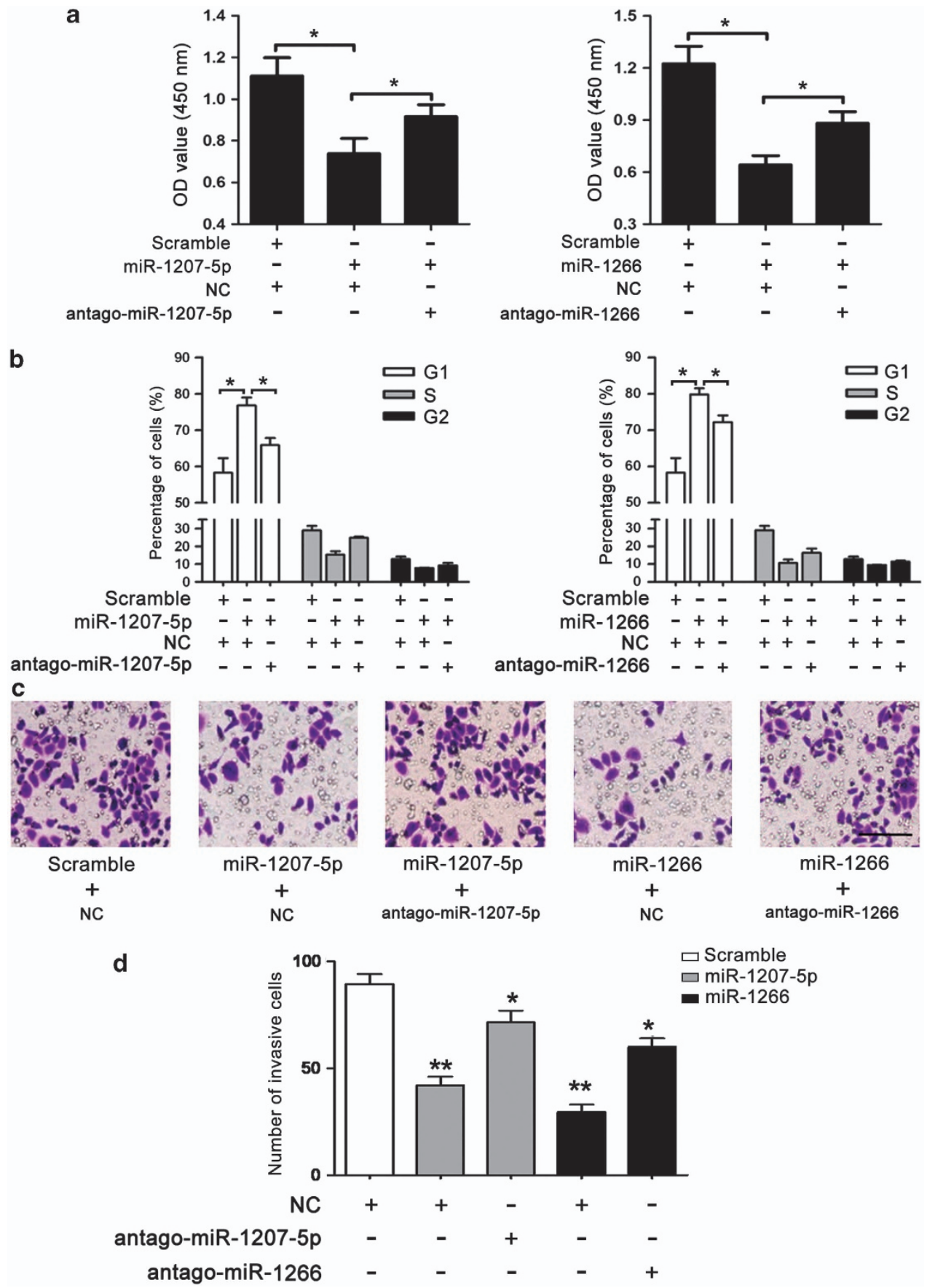

Figure 6 Inhibition of miR-1207-5p and miR-1266 rescues their anti-tumor phenotype. After SGC-7901 cells infected with the lentivirus were seeded into 96 wells or 6 wells for $18 \mathrm{~h}$, specific antago-miR and negative control (NC) were added into the wells for $96 \mathrm{~h}$, then CCK-8 assays for cell proliferation (a), flow cytometry for cell cycle (b) and transwell for cell invasion (c and $\mathbf{d}$ ) were performed ( ${ }^{*} P<0.05$, ${ }^{* \star} P<0.01$, one-way ANOVA)

In this study, we focused on targeting hTERT at the posttranscriptional level. Our study is the first to identify miRNAs that target hTERT in gastric cancer, and miR-1207-5p and miR-1266 were shown to directly suppress hTERT expression. To date, other studies have identified two different miRNAs that affect hTERT expression. A previous report showed that miR-138 directly repressed hTERT expression in anaplastic thyroid carcinomas. ${ }^{22}$ However, we found that forced expression of miR-138 downregulated hTERT expression in SGC-7901 cells without influencing the luciferase of the hTERT $3^{\prime}$ UTR. We suggest that (at least) two probable mechanisms to explain these findings exist. First, although most studies have demonstrated that miRNAs bind to the $3^{\prime}$ UTRs of their target genes, a growing body of evidence has expanded the scope of functional miRNAmRNA interaction regions to include target-gene coding regions as well. ${ }^{23}$ Therefore, we speculate that miR-138, in addition to miR-491-5p and miR-1182, may interact with the 


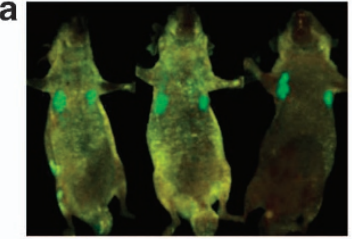

Left: Scramble Right: miR-1207-5p

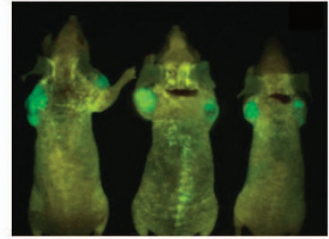

Left: Scramble Right: miR-1266
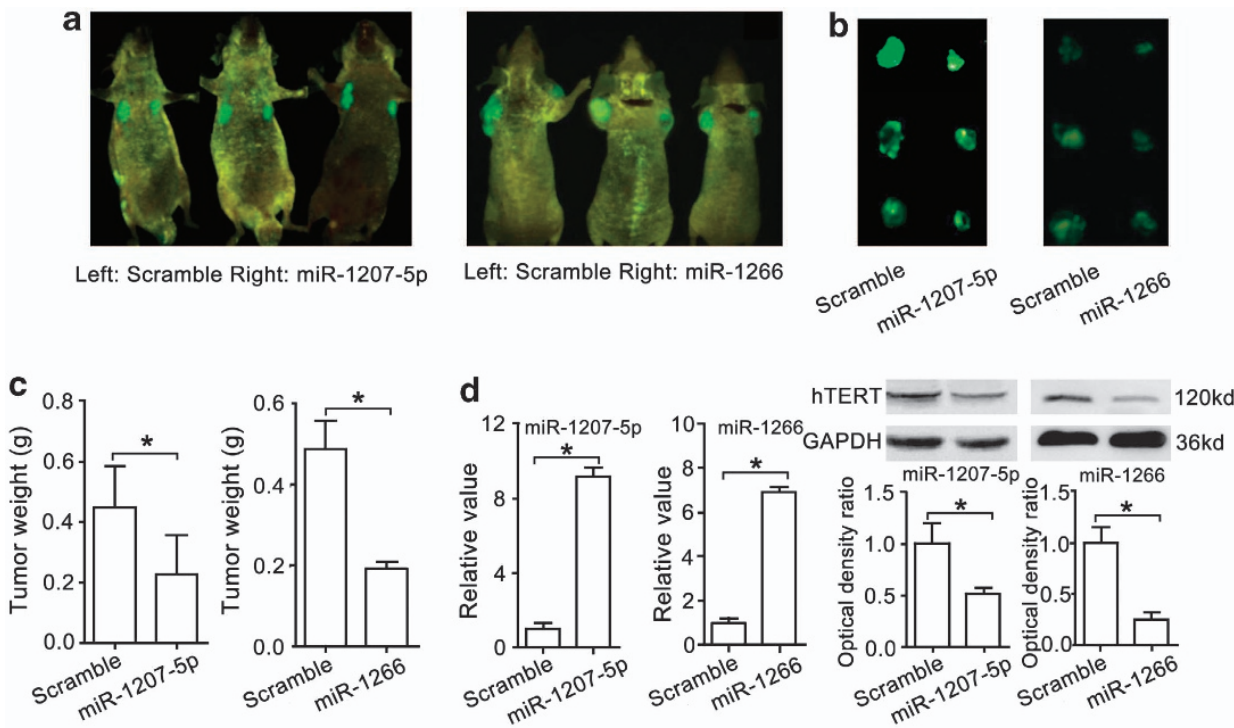

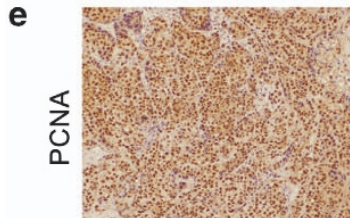

Scramble

f

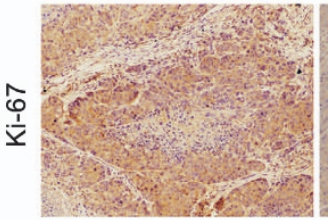

Scramble

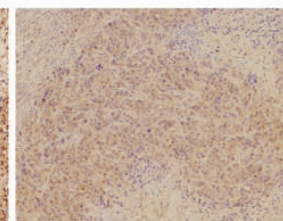

miR-1207-5p

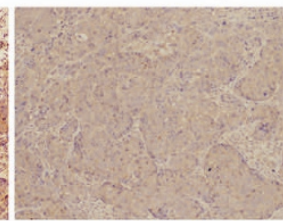

miR-1207-5p

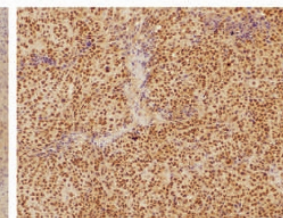

Scramble

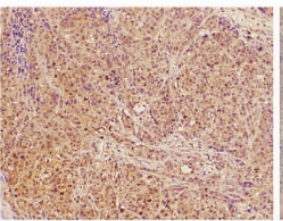

Scramble

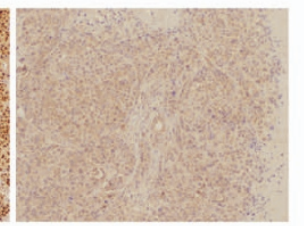

miR-1266

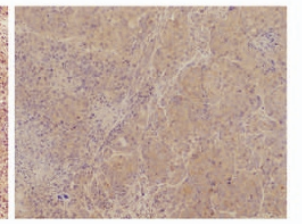

miR-1266

Figure 7 Both miR-1207-5p and miR-1266 inhibit the growth of transplanted SGC-7901 cells in nude mice. SGC-7901 cells $\left(3 \times 10^{6}\right)$ were injected s.c. into the left and right axillae of nude mice. When the tumors reached $\sim 100 \mathrm{~mm}^{3}$ in size (8 days after the injection of tumor cells), $0.1 \mathrm{ml}$ Lv-miR-1266/1207-5p or Lv-cel vector suspension $\left(10^{7} \mathrm{pfu} / \mathrm{ml}\right.$ ) was injected intra-tumorally into the center of the tumor masses. Six weeks later, the tumors were visualized and harvested. Three mice were used for each group. (a) The tumors in vivo were photographed with an In-Vivo Imaging System. Left: Lv-cel vs. miR-1207-5p groups; right: Lv-cel vs. Lv-miR-1266 groups. (b) The harvested transplanted tumors. (c) The weight of the harvested transplanted tumors. (d) The expression of miRNAs and hTERT in the tumors revealed by real-time RT-PCR (left panel) or western blot (right panel) respectively. The optical density ratio refers to mean value of the target gene/GAPDH ratio from three independent experiments. (e and $\mathbf{f}$ ) Immunohistochemistry for cell proliferation markers of PCNA (e) and Ki-67 (f), respectively

coding regions of the hTERT mRNA. Furthermore, as a variety of transcription factors bind to hTERT promoter region, these miRNAs may also affect the regulation of these transcription factors, eventually leading to the inhibition of hTERT expression. Wang et al. ${ }^{24}$ showed that miR-21 upregulates STAT3-mediated hTERT expression, thereby promoting glioblastoma cell growth. We can conclude from the above studies that one miRNA perhaps affects the expression of the same gene via different mechanisms in different cancers.

In 58 pairs of clinical gastric cancer tissues and matched corresponding adjacent tissues, we found that miR-1182, miR-1207-5p and miR-1266 were significantly downregulated in the cancer tissues, whereas miR-138 and miR-491-5p were not differentially expressed in the cancerous tissues (Supplementary Figure 2). These data suggest that the loss of miR-1182, miR-1207-5p or miR-1266 may contribute to elevated hTERT protein expression and promote the development of gastric cancer. However, only miR-1266, but not the other four miRNAs (miR-138, miR-491-5p, miR1182, miR-1207-5p), is positively correlated with better prognosis for gastric patients.

In tumor xenograft models, many miRNAs have been shown to promote gastric tumor growth, including miR-409-3p, ${ }^{25,29}$ miR-223, ${ }^{26}$ miR- $663,{ }^{27}$ miR-27a ${ }^{28}$ and miR-150. ${ }^{29}$ Conversely, several miRNAs have been shown to inhibit tumor growth in vivo, including miR-148a, ${ }^{30}$ miR-622, ${ }^{31}$ miR-148b ${ }^{32}$ and miR-126. ${ }^{33}$ However, few of these studies involved microarray analyses, general expression patterns in cancer cell lines or clinical relativity at the same time. In our study, the ectopic expression of miR-1266 and miR-1207-5p inhibited the growth and invasion of gastric cancer cells, and the delivery of miR-1266 and miR-1207-5p into transplanted tumors in nude mice significantly reduced tumor growth. These data suggest that the delivery of miR-1266 and miR-1207-5p could represent a novel therapeutic strategy 
for the treatment of gastric cancer. Papagregoriou et al. ${ }^{34}$ demonstrated that miR-1207-5p directly represses heparinbinding epidermal growth factor (HBEGF) expression and is associated with disease severity in C3-glomerulopathy. Ichihara et al. ${ }^{35}$ reported that serum miR-1266 level was considerably higher in psoriasis patients than in healthy control subjects. Our studies are the first to reveal a role for these miRNAs as cancer suppressors, and the discovery that miR-1207-5p and miR-1266 target hTERT may provide novel insights into their roles in C3-glomerulopathy. In this study, miRNA interactions were predicted using only the TargetScan website; however, as more bioinformatic tools emerge, such as MicroCosm, Microrna, RNA22, MultiMiTar, ${ }^{36}$ and PmirP, ${ }^{37}$ other potential hTERT-regulating miRNAs may be discovered.

In addition, the other three miRNAs we identified that downregulated hTERT expression (miR-138, miR-491-5p and miR-1182) also inhibited SGC-7901 cell proliferation and invasion and induced cell cycle arrest (Supplementary Figure 3), suggesting that the delivery of these miRNAs into tumors may also be an effective therapy for gastric cancer. These three miRNAs suppressed hTERT expression without binding to the $3^{\prime}$ UTR of the hTERT mRNA. Therefore, the mechanisms underlying how these miRNAs affect hTERT expression require further study.

Taken together, our results show that miR-1266 and miR-1207-5p expression levels might serve as novel biomarkers for gastric cancer and that these miRNAs exert their functions in gastric cancer cells by suppressing hTERT expression. These findings further elucidate the molecular mechanisms involved in gastric cancer tumorigenesis and development, and our results indicate that the delivery of miR-1207-5p and miR-1266 may be an effective treatment for gastric cancer.

\section{Materials and methods}

Ethics statement. All experimental procedures were approved by the Institutional Review Board of the Third Military Medical University. Written informed consent was obtained for all patient samples.

Human tissue samples and gastric cancer cell lines. All gastric tumor tissues and corresponding adjacent tissues were collected from 58 patients with gastric cancer at Southwest Hospital (during 2010 and 2011), which was approved by the Institutional Review Board of the Third Military Medical University. Gastric cancer diagnosis was made based on HE staining, immunophenotype. Information on the following was collected: age, sex, differentiation, metastasis, TNM stages, tumor size and depth of invasion. The clinical pathologic details of these 58 patients are shown in Supplementary Table 1. The following cell lines were from the American Type Culture Collection and cultured according to the vendor's instructions: U2OS, KATO-III and HEK293 cells. HF, BGC823, MKN28 and SGC-7901 cells were from Type Culture Collection of the Chinese Academy of Sciences (Shanghai, China).

Immunohistochemistry. The slides were incubated with an anti-hTERT primary antibody (R\&D Systems, Minneapolis, MN, USA). The primary antibodies were detected with biotinylated goat anti-rabbit or goat anti-mouse IgG (Vector Laboratories, Burlingame, CA, USA) secondary antibodies. Immunoreactivity was detected using a horseradish peroxidase $\left(3^{\prime}-, 3^{\prime}\right.$-diaminobenzidine) kit (BioGenex, San Francisco, CA, USA), followed by counterstaining with hematoxylin, dehydration and mounting. Antibody against Ki-67 and PCNA were from Bioword (Louis Park, MN, USA).

miRNA microarray analyses and prediction of hTERT-regulating miRNAs. miRNA was isolated using a mirVANA PARIS RNA isolation kit (Ambion, Austin, TX, USA) according to the manufacturer's instructions and the concentration was determined by $260 / 280 \mathrm{~nm}$ absorbance using a Nanodrop
ND-1000 spectrophotometer (Thermo Scientific, Wilmington, DE, USA). miRNA microarray analysis performed by the KangChen Corporation (Beijing, China) was used to compare the miRNA expression profiles in one pair of an hTERT-positive gastric cancer tissue and an hTERT-negative corresponding adjacent tissues. Then, we used TargetScan to predict miRNAs with the potential to interact with hTERT.

Detection of miRNAs using real-time RT-PCR. cDNA was synthesized using Moloney Murine Leukemia Virus reverse transcriptase (Invitrogen, Carlsbad, CA, USA), and then all the real-time RT-PCR were performed with iQ SYBR Green Supermix (BIO-RAD, Richmond, CA, USA). The housekeeping gene U6 was used as an internal control.

Construction of the luciferase reporter. To generate the luciferase reporter plasmid carrying the hTERT $3^{\prime}$-UTR, we amplified the 565 bp hTERT $3^{\prime}$-UTR from CDNA using the PCR primers $5^{\prime}$-ATCGGTGACAGTCACTAG- $3^{\prime}$ and $5^{\prime}$-TACGGATAAACAGTAGCA- $3^{\prime}$. The amplified fragment was cloned into the Spel and Hindlll sites of the pMIR-REPORT (Applied Biosystems, Foster City, CA, USA). The pMIR-REPORT vectors containing mutated versions of the hTERT $3^{\prime}$ UTR were generated from the wild-type construct with the QuikChange Site-Directed Mutagenesis Kit (Stratagene, La Jolla, CA, USA). The mutated pMIR-REPORT reporter vectors are referred to as pMIR-1207-5p M1 and pMIR-1266 M2.

Dual-luciferase assay. Wild-type or mutated versions of Luc-hTERT-3'UTR, together with the pRL-TK vector plasmid (Promega, Madison, WI, USA), were cotransfected into HEK293 cells with either an hTERT-regulating miRNA mimic or an miRNA negative control oligonucleotide (Scramble) with lipofectamine 2000 (Invitrogen). In the recovery experiments, wild-type Luc-hTERT-3' UTR, together with the pRL-TK vector plasmid, were cotransfected into HEK293 cells with either an hTERT-regulating miRNA mimic or antago-miR by lipofectamine 2000 (Invitrogen). The cells were harvested $48 \mathrm{~h}$ after transfection. Luciferase activity was determined using a Dual-luciferase assay system (Promega) according to the manufacturer's instructions. Firefly luciferase activity was normalized against Renilla luciferase activity.

Western blotting analysis. Both the hTERT and GAPDH antibodies were from Abcam (Cambridge, MA, USA). The signals were detected with the ECL Western blotting analysis system (Amersham Biosciences, Piscataway, NJ, USA), as described previously. ${ }^{38}$

Design of miRNA oligonucleotides, lentiviral constructs and cell infection. All miRNA mimic oligonucleotides and antago-miR were purchased from RiboBio (Guangzhou, China). Lentiviral constructs containing the candidate miRNAs were from GeneChem Management Inc. (Shanghai, China). Precursor miRNA expression constructs were prepared using a human immunodeficiency virus (HIV)-based lentiviral vector system. The packaged lentiviruses are referred to as LV-miR-138, LV-miR-491-5p, LV-miR-1182, LV-miR1207-5p, LV-miR-1266 and LV-Scramble. These viruses, as well as the lentiviral to knockdown hTERT expression (si hTERT), were used to infect SGC-7901 cells at a multiplicity of infection (MOI) of 10; the infection efficiency was $100 \%$.

Cell proliferation and cell cycle assays. Relative cell growth was determined using the Cell Counting Kit-8 (CCK-8, Dojindo, Kumamoto, Japan) assay, as described previously. ${ }^{39}$ For the cell cycle analyses, cells were plated into six-well plates and flow cytometry was performed $48 \mathrm{~h}$ later, as described previously. ${ }^{40}$

Invasion assay. The effect of hTERT-regulating miRNAs on the invasive abilities of SGC-7901 cells was determined using matrigel-coated Matrigel Invasion Chambers ( $8 \mu \mathrm{m}$ pore size, BD Biosciences, Franklin Lakes, NJ, USA). Cells infected with lentivirus described above were seeded into chambers with serum-free medium (to monitor cell growth at lowest rapid). Subsequently, the cells were transfected with antago-miR or pIRES2 for certain hours, the chambers were then transferred to wells filled with culture medium containing $10 \% \mathrm{FBS}$. After $48 \mathrm{~h}$ of incubation, invasive cells on the bottom were fixed and stained with $0.05 \%$ crystal violet. The number of invasive cells from five random areas of the membrane was counted with light microscopy.

Gastric cancer xenografts. The studies were performed in accordance with the NIH Animal Use Guidelines and a protocol approved by the SIU Animal Care Committee. SGC-7901 cells $\left(3 \times 10^{6}\right)$ in $100 \mu$ l medium were injected 
subcutaneous (s.c.) into the left and right axillae of female nude mice, as previously described. ${ }^{41}$ When the tumors reached $100 \mathrm{~mm}^{3}$ in size ( $\sim 8$ days after

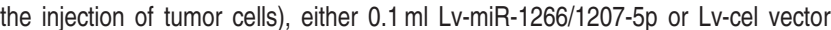
suspension $\left(10^{7} \mathrm{pfu} / \mathrm{ml}\right)$ was injected intra-tumorally into the center of the tumor mass. After 6 weeks, the tumors were visualized with an In-Vivo Imaging System (Cambridge Research \& Instrumentation, MA, USA). Then, the tumors were harvested to evaluate their weight, miRNA expression and hTERT expression. Three mice were used for each group.

Statistical analysis. All data are presented as the means \pm S.E. and were analyzed with Prism 5.0 software (GraphPad, San Diego, CA, USA). The pairedsamples $t$-test was used to assay the differences in the miRNA expression levels between cancer tissues and corresponding adjacent tissues. The independentsamples $t$-test is used for two groups, while one-way ANOVA is used for three or more groups. Kaplan-Meier's method was used for survival analysis. $P<0.05$ was considered statistically significant.

\section{Conflict of Interest}

The authors declare no conflict of interest.

Acknowledgements. This study was supported by grants from the National Nature Science Foundation of China (No. 30900679), Chongqing Science Fund for Distinguished Young Scholars (CSTC, 2009BA5045), Chongqing Natural Science Foundation (cstcjja10054).

1. Noel JF, Wellinger RJ. Exposing secrets of telomere-telomerase encounters. Cell 2012; 150: 453-454.

2. Nandakumar J, Cech TR. Finding the end: recruitment of telomerase to telomeres. Nat Rev Mol Cell Biol 2013; 14: 69-82.

3. Cifuentes-Rojas C, Shippen DE. Telomerase regulation. Mutat Res 2012; 730: 20-27.

4. Zhang Y, Chen X, Xu X, Wang X, Yuan G, Sun D et al. Knockdown of hTERT alters biophysical properties of $\mathrm{K} 562$ cells resulting in decreased migration rate in vitro. Cell Biochem Biophys 2011; 61: 595-603.

5. Lu MH, Deng JQ, Cao YL, Fang DC, Zhang Y, Yang SM. Prognostic role of telomerase activity in gastric adenocarcinoma: A meta-analysis. Exp Ther Med 2012; 3: 728-734.

6. Horikawa I, Barrett JC. Transcriptional regulation of the telomerase hTERT gene as a target for cellular and viral oncogenic mechanisms. Carcinogenesis 2003; 24: 1167-1176.

7. Toffanin S, Villanueva A, Llovet JM. miRNA delivery: emerging therapy for hepatocellular carcinoma. Gastroenterology 2010; 138: 1202-1204.

8. Sprouse AA, Steding CE, Herbert BS. Pharmaceutical regulation of telomerase and its clinical potential. J Cell Mol Med 2012; 16: 1-7.

9. Liu X, Inomata M, Ogawara T, Saneyoshi M, Yamaguchi T. Telomere shortening in human $\mathrm{HL} 60$ cells by treatment with $3^{\prime}$-azido- $2^{\prime}, 3^{\prime}$-dideoxynucleosides and telomerase inhibition by their $5^{\prime}$-triphosphates. Nucleosides Nucleotides Nucleic Acids 2007; 26 : 1067-1071.

10. Rossi A, Russo G, Puca A, La Montagna R, Caputo M, Mattioli E et al. The antiretroviral nucleoside analogue Abacavir reduces cell growth and promotes differentiation of human medulloblastoma cells. Int J Cancer 2009; 125: 235-243.

11. Gryaznov SM. Oligonucleotide $n 3^{\prime}->p 5^{\prime}$ phosphoramidates and thio-phoshoramidates as potential therapeutic agents. Chem Biodivers 2010; 7: 477-493.

12. Mahendar P, Sirisha K, Kulandaivelu U, Shankar PL, Radhika T, Sadanandam A. In silico evaluation of TERT inhibition by anticancer drugs. J Mol Model 2012; 18: 4739-4744.

13. Chakraborty S, Ghosh U, Bhattacharyya NP, Bhattacharya RK, Roy M. Inhibition of telomerase activity and induction of apoptosis by curcumin in K-562 cells. Mutat Res 2006; 596: 81-90.

14. Vonderheide RH, Domchek SM, Schultze JL, George DJ, Hoar KM, Chen DY et al Vaccination of cancer patients against telomerase induces functional antitumor CD8 + T lymphocytes. Clin Cancer Res 2004; 10: 828-839.

15. Brunsvig PF, Aamdal S, Gjertsen MK, Kvalheim G, Markowski-Grimsrud CJ, Sve I et al. Telomerase peptide vaccination: a phase $1 / / l$ study in patients with non-small cell lung cancer. Cancer Immunol Immunother 2006; 55: 1553-1564.

16. Yang SM, Fang DC, Yang JL, Chen L, Luo YH, Liang GP. Antisense human telomerase reverse transcriptase could partially reverse malignant phenotypes of gastric carcinoma cell line in vitro. Eur J Cancer Prev 2008; 17: 209-217.

17. Chen $L$, Liang GP, Tang XD, Chen $T$, Cai $Y G$, Fang DC et al. In vitro anti-tumor immune response induced by dendritic cells transfected with hTERT recombinant adenovirus. Biochem Biophys Res Commun 2006; 351: 927-934.
18. Chen L, Tang XD, Yu ST, Ai ZH, Fang DC, Cai YG et al. Induction of anti-tumour immunity by dendritic cells transduced with hTERT recombinant adenovirus in mice. J Pathol 2009; 217: 685-692.

19. Yu ST, Chen L, Wang HJ, Tang XD, Fang DC, Yang SM. hTERT promotes the invasion of telomerase-negative tumor cells in vitro. Int J Oncol 2009; 35: 329-336.

20. Zhang RG, Zhao JJ, Yang LQ, Yang SM, Wang RQ, Chen WS et al. RNA interferencemediated $\mathrm{hTERT}$ inhibition enhances TRAIL-induced apoptosis in resistant hepatocellular carcinoma cells. Oncol Rep 2010; 23: 1013-1019.

21. Yu ST, Li C, Lu MH, Liang GP, Li N, Tang XD et al. Noninvasive and real-time monitoring of the therapeutic response of tumors in vivo with an optimized hTERT promoter. Cancer-Am Cancer Soc 2012; 118: 1884-1893.

22. Mitomo S, Maesawa C, Ogasawara S, Iwaya T, Shibazaki M, Yashima-Abo A et al. Downregulation of miR-138 is associated with overexpression of human telomerase reverse transcriptase protein in human anaplastic thyroid carcinoma cell lines. Cancer Sci 2008; 99: 280-286.

23. Kang JG, Majerciak V, Uldrick TS, Wang X, Kruhlak M, Yarchoan R et al. Kaposi's sarcoma-associated herpesviral IL- 6 and human IL-6 open reading frames contain miRNA binding sites and are subject to cellular miRNA regulation. J Pathol 2011; 225: 378-389.

24. Wang YY, Sun G, Luo H, Wang XF, Lan FM, Yue X et al. MiR-21 modulates hTERT through a STAT3-dependent manner on glioblastoma cell growth. Cns Neurosci Ther 2012; 18: 722-728.

25. Li C, Nie H, Wang M, Su L, Li J, Yu B et al. MicroRNA-409-3p regulates cell proliferation and apoptosis by targeting PHF10 in gastric cancer. Cancer Lett 2012; 320: 189-197.

26. Li J, Guo Y, Liang X, Sun M, Wang G, De W et al. MicroRNA-223 functions as an oncogene in human gastric cancer by targeting FBXW7/hCdc4. J Cancer Res Clin Oncol 2012; 138: 763-774.

27. Yi C, Wang $Q$, Wang $L$, Huang $Y$, Li L, Liu L et al. MiR-663, a microRNA targeting p21(WAF1/CIP1), promotes the proliferation and tumorigenesis of nasopharyngeal carcinoma. Oncogene 2012; 31: 4421-4433.

28. Zhao XH, Yang L, Hu JG. Down-regulation of miR-27a might inhibit proliferation and drug resistance of gastric cancer cells. J Exp Clin Canc Res 2011; 30: 55.

29. Wu Q, Jin H, Yang Z, Luo G, Lu Y, Li K et al. MiR-150 promotes gastric cancer proliferation by negatively regulating the pro-apoptotic gene EGR2. Biochem Biophys Res Commun 2010; 392: 340-345.

30. Zheng B, Liang L, Wang C, Huang S, Cao X, Zha R et al. MicroRNA-148a suppresses tumor cell invasion and metastasis by downregulating ROCK1 in gastric cancer. Clin Cancer Res 2011; 17: 7574-7583.

31. Guo XB, Jing CQ, Li LP, Zhang L, Shi YL, Wang JS et al. Down-regulation of miR-622 in gastric cancer promotes cellular invasion and tumor metastasis by targeting ING1 gene. World J Gastroenterol 2011; 17: 1895-1902.

32. Song YX, Yue ZY, Wang ZN, Xu YY, Luo Y, Xu HM et al. MicroRNA-148b is frequently down-regulated in gastric cancer and acts as a tumor suppressor by inhibiting cell proliferation. Mol Cancer 2011; 10: 1.

33. Feng RH, Chen XH, Yu YY, Su LP, Yu BQ, Li JF et al. miR-126 functions as a tumour suppressor in human gastric cancer. Cancer Lett 2010; 298: 50-63.

34. Papagregoriou G, Erguler K, Dweep H, Voskarides K, Koupepidou P, Athanasiou Y et al. A miR-1207-5p binding site polymorphism abolishes regulation of HBEGF and is associated with disease severity in CFHR5 nephropathy. PloS One 2012; 7: e31021.

35. Ichihara A, Jinnin M, Oyama R, Yamane K, Fujisawa A, Sakai K et al. Increased serum levels of miR-1266 in patients with psoriasis vulgaris. Eur J Dermatol 2012; 22: 68-71.

36. Mitra R, Bandyopadhyay S. MultiMiTar: a novel multi objective optimization based miRNA-target prediction method. PloS One 2011; 6: e24583.

37. Zhao D, Wang Y, Luo D, Shi X, Wang L, Xu D et al. PMirP: a pre-microRNA prediction method based on structure-sequence hybrid features. Artif Intell Med 2010; 49: 127-132.

38. Yu ST, Yang YB, Liang GP, Li C, Chen L, Shi CM et al. An optimized telomerase-specific lentivirus for optical imaging of tumors. Cancer Res 2010; 70: 2585-2594.

39. Wang C, Gao DM, Guo K, Kang XN, Jiang K, Sun C et al. Novel synergistic antitumor effects of rapamycin with bortezomib on hepatocellular carcinoma cells and orthotopic tumor model. Bmc Cancer 2012; 12: 166.

40. Xiong Z, Lu MH, Fan YH, Cao YL, Hu CJ, Wu YY et al. Downregulation of heparanase by RNA interference inhibits invasion and tumorigenesis of hepatocellular cancer cells in vitro and in vivo. Int J Oncol 2012; 40: 1601-1609.

41. Esquela-Kerscher A, Trang P, Wiggins JF, Patrawala L, Cheng A, Ford L et al. The let-7 microRNA reduces tumor growth in mouse models of lung cancer. Cell Cycle 2008; 7: 759-764.

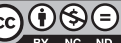

Cell Death and Disease is an open-access journal published by Nature Publishing Group. This work is licensed under a Creative Commons Attribution-NonCommercialNoDerivs 3.0 Unported License. To view a copy of this license, visit http://creativecommons.org/licenses/by-nc-nd/3.0/ 